\title{
Communication Efficient Secret Sharing
}

\author{
Wentao Huang${ }^{1}$, Michael Langberg ${ }^{2}$, Joerg Kliewer ${ }^{3}$, and Jehoshua Bruck ${ }^{1}$ \\ ${ }^{1}$ California Institute of Technology \\ ${ }^{2}$ State University of New York at Buffalo \\ ${ }^{3}$ New Jersey Institute of Technology
}

\begin{abstract}
A secret sharing scheme is a method to store information securely and reliably. Particularly, in the threshold secret sharing scheme (due to Shamir), a secret is divided into shares, encoded and distributed to parties, such that any large enough collection of parties can decode the secret, and a smaller (then threshold) set of parties cannot collude to deduce any information about the secret. While Shamir's scheme was studied for more than 35 years, the question of minimizing its communication bandwidth was not considered. Specifically, assume that a user (or a collection of parties) wishes to decode the secret by receiving information from a set of parties; the question we study is how to minimize the total amount of communication between the user and the parties. We prove a tight lower bound on the amount of communication necessary for decoding, and construct secret sharing schemes achieving the bound. The key idea for achieving optimal communication bandwidth is to let the user receive information from more than the necessary number of parties. In contrast, the current paradigm in secret sharing schemes is to decode from a minimum set of parties. Hence, existing secret sharing schemes are not optimal in terms of communication bandwidth. In addition, we consider secure distributed storage where our proposed communication efficient secret sharing schemes improve disk access complexity during decoding.
\end{abstract}

\section{Introduction}

Consider the scenario that $n$ parties wish to store a secret securely and reliably. To this end, a dealer will distribute the secret into $n$ shares, i.e., one share for each party, such that 1) (reliability) a collection $\mathcal{A}$ of "authorized" subsets of the parties can reconstruct the secret, and 2) (security) a collection $\mathcal{B}$ of "blocked" subsets of the parties cannot collude to deduce any information about the secret. A scheme to distribute the secret into shares with respect to access structure $(\mathcal{A}, \mathcal{B})$ is called a secret sharing scheme, initially studied in the seminal works by Shamir [13] and Blakley [2]. Besides its application in distributed storage of secret data, secret sharing became a fundamental cryptographic primitive and is used 
as a building block in numerous secure protocols, most notably in threshold cryptography and secure multi-party computation [1].

We focus on secret sharing schemes for the threshold access structure, i.e., $\mathcal{A}$ contains all subsets of $\{1, \ldots, n\}$ of size at least $n-r$, and $\mathcal{B}$ contains all subsets of $\{1, \ldots, n\}$ of size at most $z$. In other words, the secret can be reconstructed in the loss of any $r$ parties, and any $z$ parties cannot collude to deduce any information about the secret. The threshold access structure is particularly important in practice, because for this case, space and computationally efficient secret sharing schemes are known. Specifically, Shamir [13] constructs an elegant and efficient threshold scheme using the idea of polynomial interpolation, for the case of $z=n-r-1$. Shamir's scheme is later shown to be closely related to Reed-Solomon codes and is generalized to the case of arbitrary $z$ [11]. Shamir's scheme and the generalized scheme [11] achieve optimal usage of storage space, in the sense that fixing the size of the shares, the schemes store a secret of maximum size. The schemes are computationally efficient as decoding the secret is equivalent to decoding ReedSolomon codes. An example of Shamir's scheme is shown in Figure 1. Other generalization and extension of Shamir's scheme may be found in $[9,5,15,10]$. The reader is also referred to [1] for an up-to-date survey on secret sharing.

\begin{tabular}{c|c|c} 
Party 1 & Party 2 & Party 3 \\
\hline$m+k$ & $m+2 k$ & $m+3 k$
\end{tabular}

Figure 1: Shamir's scheme for $n=3, r=1, z=1$. Operations are over $\mathbb{F}_{7}$. The scheme stores a secret of one symbol, denoted by $m$. $k$ is a uniformly and independently distributed random variable. Note that the symbol stored by any single party is independent of the secret, and that the secret can be recovered from the symbols stored by any two parties.

In addition to space and computational efficiency, this paper introduces communication efficiency for secret sharing schemes. Consider the scenario that a user wishes to decode the secret by downloading information from the parties that he has access to. The question is, what is the minimum amount of information to be communicated. It is of practical interest to design secret sharing schemes that require communicating only a small amount of information during decoding, because decoding will be completed in a more timely manner and communication resource will be more efficiently utilized.

For existing secret sharing schemes, e.g., $[13,11,9,5,15,10]$, a common practice in decoding is that the user will communicate with a minimum set of parties, i.e., exactly $n-r$ parties (even if he has access to more parties) and download the whole share stored by these parties. We show that this paradigm is not optimal in terms of communication efficiency, demonstrated by the following motivating examples. 


\begin{tabular}{c|c|c} 
Party 1 & Party 2 & Party 3 \\
\hline$m_{1}+k_{1}$ & $m_{1}+2 k_{1}$ & $m_{1}+3 k_{1}$ \\
$m_{2}+k_{2}$ & $m_{2}+2 k_{2}$ & $m_{2}+3 k_{2}$
\end{tabular}

(a) Shamir's Scheme

\begin{tabular}{c|c|c} 
Party 1 & Party 2 & Party 3 \\
\hline$m_{1}+m_{2}+k_{1}$ & $m_{1}+2 m_{2}+4 k_{1}$ & $m_{1}+3 m_{2}+2 k_{1}$ \\
$m_{1}+4 m_{2}+2 k_{1}+6 k_{2}$ & $m_{1}+5 m_{2}+4 k_{1}+3 k_{2}$ & $m_{1}+6 m_{2}+k_{1}+4 k_{2}$
\end{tabular}

(b) Proposed Scheme

Figure 2: Two secret sharing schemes for $n=3, r=1$ and $z=1$ over $\mathbb{F}_{7}$. Both schemes store a secret of two symbols $\left(m_{1}, m_{2}\right)$. In both schemes, $k_{1}$ and $k_{2}$ are i.i.d. uniformly distributed random variables. The construction of scheme (b) is described later in detail in Section 4.1, Example 1. Note that in (b), if all three parties are accessible, then the secret $\left(m_{1}, m_{2}\right)$ can be decoded by communicating only the three symbols in the first row, denoted by $b_{11}, b_{12}$ and $b_{13}$. Specifically, $m_{1}=3 b_{11}+4 b_{12}+b_{13}$ and $m_{2}=b_{11}+4 b_{12}+2 b_{13}$. Also note that scheme (b) can correct one column erasure, i.e., $m_{1}, m_{2}, k_{1}$ and $k_{2}$ can be decoded as long as any two parties are accessible. For example, suppose that only Party 1 and 2 are accessible and denote by $b_{21}, b_{22}, b_{23}$ the three symbols in the second row of (b), then $m_{1}=4 b_{11}+3 b_{21}+b_{22}, m_{2}=3 b_{11}+3 b_{12}+6 b_{21}+2 b_{22}, k_{1}=b_{11}+4 b_{12}+5 b_{21}+4 b_{22}$ and $k_{2}=4 b_{11}+6 b_{12}+b_{21}+3 b_{22}$.

\subsection{Motivating Example}

Consider Shamir's scheme in the example of Figure 1, that stores one symbol securely and reliably for the setting $n=3, r=1$ and $z=1$. In order to decode the secret symbol, a user needs to download two symbols from any two parties, and therefore the amount of communication is two symbols. Now suppose the same scheme is repeated twice in order to store a secret of two symbols, as shown in Figure 2a. Then to decode the secret, the amount of communication is four symbols.

Different from Shamir's scheme, we propose another linear secret sharing scheme in Figure $2 \mathrm{~b}$ that also stores a secret of two symbols for the same setting, using the same amount of storage space. In order to decode the secret in this scheme, it suffices to communicate only the three symbols in the first row. Therefore, the amount of communication is improved to three symbols, provided that all three parties are accessible.

We use the examples in Figure 2 to highlight several ideas to reduce communication during decoding. Firstly, the amount of communication depends on the number of accessible parties. If there are exactly $n-r$ accessible parties, then the amount of communication of Shamir's scheme, 4 symbols in the example, is necessary. However, if more than $n-r$ parties are accessible, then a smaller amount of communication can be achieved. In fact 
we show that the necessary amount of communication decreases strictly as the number of accessible parties increases. Secondly, in our scheme we use the idea of distributing multiple subshares (symbols) to a party (essentially using the ideas of array codes $[4,3]$ ). In contrast, Shamir's scheme only distributes one symbol to each party except for trivial repetitions. We remark that for secret sharing schemes with general (non-threshold) access structures, it is natural to distribute multiple symbols to each party $[7,8]$, because in general different parties play different roles in the access structure and should store different amount of information. However, we show that for the threshold case in which all parties play an equal role and store the same amount of information, it is still beneficial to distribute multiple symbols to each party, for the purpose of reducing the amount of communication. Thirdly, during decoding it is not always necessary to download the complete share stored by a party. In general, a party can preprocess its share so that the user will download a function of the share.

We also remark on an interesting analog between communication efficient secret sharing and the well-studied subject of regenerating codes $[6,14,12]$. Consider a regenerating code of length $n$ that is able to corrects $r>1$ erasures. If only one erasure occurs, then compared to repairing from a minimum set of $n-r$ nodes, repairing from all the $n-1$ available nodes will significantly reduce the total amount of communication that occurs during the repair. In this sense, for both regenerating codes and communication efficient secret sharing, a key idea is to involve more available nodes/parties than the minimum set of them during repair/decoding, for the purpose of reducing the repair/decoding bandwidth.

\subsection{Results}

In Section 3, we prove a tight information-theoretic lower bound on the amount of information to be communicated during decoding, given a set of accessible parties $I \subset\{1, \ldots, n\}$. The bound implies that the necessary amount of communication decreases as $|I|$ increases. Particularly, we show that the minimum communication overhead for the case of $|I|=n$ is only a fraction $(n-r-z) /(n-z)$ of the minimum communication overhead when $|I|=n-r$.

In Section 4, we construct secret sharing schemes using the ideas described in Section 1.1. Our construction utilizes Reed-Solomon codes and achieves the lower bound on communication for all $I \in \mathcal{A}$ such that $|I|=n$ or $|I|=n-r$. Note that the case of $|I|=n$ is particularly important because it corresponds to the best case in terms of communication efficiency, i.e., all parties are accessible. Furthermore, $|I|=n$ is arguably the most relevant case for the application of distributed storage of secret data, where the storage nodes are usually highly available. In addition to achieving good communication efficiency, the construction is also efficient in terms of space and computational complexity, similar to Shamir's scheme. Specifically, our scheme achieves optimal usage of storage space, in the sense that fixing the size of the shares, a secret of maximum size is stored. The decoding algorithm can be implemented as the decoding algorithm for Reed-Solomon codes and therefore is computationally efficient. 
We then give an existence proof of the tightness of the lower bound on communication in Section 5. Specifically, we design another secret sharing scheme based on random linear codes, such that the scheme meets the security requirement deterministically, and meets the reliability requirement with high probability as the field size grows. The scheme achieves optimal communication efficiency, i.e., it achieves the lower bound on communication for all $I \in \mathcal{A}$, hence proving the tightness of the lower bound. The scheme also achieves optimal space efficiency. However, compared to the construction based on Reed-Solomon codes, this scheme requires a larger field size and is less efficient in terms of computational complexity.

Finally, in the application of secure storage, the lower bound on communication is also a lower bound on the number of symbol-reads from disks during decoding. In both of our schemes, the number of symbol-reads equals to the amount of communication. Therefore, our schemes are very efficient in terms of disk operations, which is another practical advantage over existing secret sharing schemes. In addition, by involving more than the minimum number of disks for decoding, our schemes balance the load at the disks and achieve a higher degree of parallelization.

\section{Secret Sharing}

Consider a secret $\boldsymbol{m}$ as a uniformly distributed random variable over some alphabet. For the purposes of reliability and security, it is desirable to distribute the secret into $n$ shares, so that 1) $\boldsymbol{m}$ can be recovered from any $n-r$ shares, and 2) any $z$ shares do not reveal any information about $\boldsymbol{m}$, i.e., they are statistically independent. Such a scheme is called a threshold secret sharing scheme, defined formally as follows. Let $\mathcal{Q}$ be a general $Q$-ary alphabet, i.e., $|\mathcal{Q}|=Q$. Denote by $[n]=\{1, \ldots, n\}$. For any index set $I \subset[n]$ and a vector $\boldsymbol{c}=\left(c_{1}, \ldots, c_{n}\right)$, denote by $\boldsymbol{c}_{I}=\left(c_{i}\right)_{i \in I}$.

Definition 1. An $(n, k, r, z)_{\mathcal{Q}}$ secret sharing scheme consists of a randomized encoding function $F$ that maps a secret $\boldsymbol{m} \in \mathcal{Q}^{k}$ to $\boldsymbol{c}=\left(c_{1}, \ldots, c_{n}\right)=F(\boldsymbol{m}) \in \mathcal{Q}^{n}$, such that

1) (Reliability) The secret $\boldsymbol{m}$ is completely determined by any $n-r$ shares (entries) of $\boldsymbol{c}$. This guarantees $\boldsymbol{m}$ is recoverable in the loss of any $r$ shares. Formally,

$$
H\left(\boldsymbol{m} \mid \boldsymbol{c}_{I}\right)=0, \quad \forall I \subset[n],|I|=n-r .
$$

Therefore for any $I \subset[n],|I|=n-r$, there exists a decoding function $D_{I}^{*}: \mathcal{Q}^{n-r} \rightarrow$ $\mathcal{Q}^{k}$ such that $D_{I}^{*}\left(\boldsymbol{c}_{I}\right)=\boldsymbol{m}$.

2) (Security) Any z shares of $\boldsymbol{c}$ do not reveal any information about $\boldsymbol{m}$. This guarantees $\boldsymbol{m}$ is secure if any $z$ shares are exposed to an eavesdropper. Formally,

$$
H\left(\boldsymbol{m} \mid \boldsymbol{c}_{I}\right)=H(\boldsymbol{m}), \quad \forall I \subset[n],|I|=z .
$$


The following proposition gives an upper bound on $k$, the size of the secret.

Proposition 1. For any $(n, k, r, z)_{\mathcal{Q}}$ secret sharing scheme, it follows that

$$
k \leq n-r-z
$$

Proof.

$$
\begin{aligned}
k=H(\boldsymbol{m}) & =H\left(\boldsymbol{m} \mid \boldsymbol{c}_{[z]}\right) \\
& \leq H\left(\boldsymbol{m}, \boldsymbol{c}_{[n-r]} \mid \boldsymbol{c}_{[z]}\right) \\
& =H\left(\boldsymbol{m} \mid \boldsymbol{c}_{[n-r]}, \boldsymbol{c}_{[z]}\right)+H\left(\boldsymbol{c}_{[n-r]} \mid \boldsymbol{c}_{[z]}\right) \\
& =H\left(\boldsymbol{c}_{[n-r]} \mid \boldsymbol{c}_{[z]}\right) \\
& =H\left(\boldsymbol{c}_{\{z+1, \ldots, n-r\}}\right) \leq n-r-z,
\end{aligned}
$$

where (4) follows from the security requirement, (5) follows from the chain rule, and (6) follows from the reliability requirement.

If a secret sharing scheme achieves equality in (3), then the scheme is optimal in terms of storage space usage and is said to be capacity-achieving. Capacity-achieving secret sharing schemes for the case that $z=n-r-1$ are studied in the seminal work by Shamir [13], and are later generalized to arbitrary values of $n, r$ and $z$ [11].

\section{Lower Bound on Communication Overhead}

Suppose that the $n$ shares of the secret are stored by $n$ parties or distributed storage nodes, and a user wants to decode the secret. In the standard way of decoding, by Definition 1, the user will connect to any $n-r$ nodes and retrieves one share, i.e., one $Q$-ary symbol, from each node. Therefore, by communicating $n-r$ symbols, the user can decode a secret of at most $n-r-z$ symbols. It is clear that a communication overhead of $z$ symbols occurs during decoding. The question is, whether it is possible to reduce the communication overhead. We answer this question affirmatively in the remaining part of the paper.

There are two key ideas for improving the communication overhead. Firstly, in many practical scenarios and particularly in distributed storage systems, often time more than $n-r$ nodes are available. In this case, it is not necessary to restrict the user to connect and communicate to only $n-r$ nodes. Secondly, when a user connects to a storage node, it is not necessary to communicate the complete share stored by the node. Instead, it may suffice to communicate only a part of the share or, in general, a function of the share. In other words, a node can preprocess its share before transmitting it to the user.

Motivated by these ideas, for any $I \subset[n],|I| \geq n-r$, define a class of preprocessing functions $E_{I, i}: \mathcal{Q} \rightarrow \mathcal{S}_{I, i}$, where $\left|\mathcal{S}_{I, i}\right| \leq|\mathcal{Q}|$, that maps $c_{i}$ to $e_{I, i}=E_{I, i}\left(c_{i}\right)$. Let $\boldsymbol{e}_{I}=$ $\left(e_{I, i}\right)_{i \in I}$, and define a class of decoding functions $D_{I}: \prod_{i \in I} \mathcal{S}_{I, i} \rightarrow \mathcal{Q}^{k}$, such that $D_{I}\left(\boldsymbol{e}_{I}\right)=$ 
$\boldsymbol{m}$. For a naive example, consider any $I$ such that $|I|=n-r$. Then for $i \in I$, we may let $\mathcal{S}_{I, i}=\mathcal{Q}$, let $E_{I, i}$ be the identity function, and let $D_{I}$ be the naive decoding function $D_{I}^{*}$ described in Definition 1. In the remaining paper, when $I$ is clear from the context, we will suppress it in the subscripts of $\mathcal{S}_{I, i}, E_{I, i}$ and $e_{I, i}$, and denote them by $\mathcal{S}_{i}, E_{i}$ and $e_{i}$ instead. We now formally define the notion of communication overhead in decoding. Note that all log functions in the paper are base $Q$.

Definition 2. For any I such that $|I| \geq n-r$, define the communication overhead function to be $\mathrm{CO}(I)=\sum_{i \in I} \log \left|\mathcal{S}_{I, i}\right|-k$. Namely, $\mathrm{CO}(I)$ is the amount of extra information, measured in $Q$-ary symbols, that one needs to communicate in order to decode a secret of $k$ symbols, provided that the set of accessible shares is indexed by $I$.

The following result provides a lower bound on the communication overhead function.

Theorem 1. For any $(n, k, r, z)_{\mathcal{Q}}$ secret sharing scheme with preprocessing functions $\left\{E_{I, i}\right\}_{i \in[n],|I| \geq n-r}$ and decoding functions $\left\{D_{I}\right\}_{|I| \geq n-r}$, it follows that

$$
\mathrm{CO}(I) \geq \frac{k z}{|I|-z}
$$

Proof. Consider arbitrary $I=\left\{i_{1}, \ldots, i_{|I|}\right\}$ such that $|I| \geq n-r$. Assume without loss of generality that $\left|\mathcal{S}_{i_{1}}\right| \leq\left|\mathcal{S}_{i_{2}}\right| \leq \ldots \leq\left|\mathcal{S}_{i_{|I|} \mid}\right|$. Recall that $\boldsymbol{e}_{I}=\left(e_{i_{1}}, \ldots, e_{i_{\mid} I \mid}\right)$ is the output of the preprocessing functions.

$$
\begin{aligned}
H\left(e_{i_{1}}, \ldots, e_{i_{|I|-z}}\right) & \stackrel{(a)}{\geq} H\left(e_{i_{1}}, \ldots, e_{i_{|I|-z}} \mid e_{i_{|I|-z+1}}, \ldots, e_{i_{|I|}}\right) \\
& \stackrel{(b)}{=} H\left(e_{i_{1}}, \ldots, e_{i_{|I|-z}} \mid e_{i_{|I|-z+1}}, \ldots, e_{i_{|I|}}\right)+H\left(\boldsymbol{m} \mid e_{i_{1}}, \ldots, e_{i_{|I|}}\right) \\
& \stackrel{(c)}{=} H\left(\boldsymbol{m}, e_{i_{1}}, \ldots, e_{i_{|I|-z}} \mid e_{i_{|I|-z+1}}, \ldots, e_{i_{|I|}}\right) \\
& \geq H\left(\boldsymbol{m} \mid e_{i_{|I|-z+1}}, \ldots, e_{i_{|I|}}\right) \\
& \stackrel{(d)}{=} H(\boldsymbol{m})=k,
\end{aligned}
$$

where (a) follows from conditioning reduces entropy, (b) follows from (1), (c) follows form the chain rule, and (d) follows from (2). Therefore it follows from (8) that

$$
\prod_{j=1}^{|I|-z}\left|\mathcal{S}_{i_{j}}\right| \geq q^{H\left(e_{i_{1}}, \ldots, e_{i|I|-z}\right)} \geq q^{k}
$$

and so

$$
\sum_{j=1}^{|I|-z} \log \left|\mathcal{S}_{i_{j}}\right| \geq k
$$


It then follows from $\left|\mathcal{S}_{i_{1}}\right| \leq \ldots \leq\left|\mathcal{S}_{i_{|I|}}\right|$ that,

$$
\log \left|\mathcal{S}_{i_{|I|-z}}\right| \geq \frac{k}{|I|-z}
$$

and that,

$$
\log \left|\mathcal{S}_{i_{|I|-z+j}}\right| \geq \log \left|\mathcal{S}_{i_{|I|-z} \mid}\right| \geq \frac{k}{|I|-z}, \quad j=1, \ldots, z
$$

Combining (9) and (10) we have,

$$
\mathrm{CO}(I)=\sum_{j=1}^{|I|} \log \left|\mathcal{S}_{i_{j}}\right|-k \geq \frac{k z}{|I|-z} .
$$

For capacity achieving schemes, i.e., schemes with $k=n-r-z$, Theorem 1 implies that if $|I|=n-r$, then the communication overhead is at least $z$. Notice that the naive decoding function $D_{I}^{*}$ in Definition 1 achieves this bound on communication overhead. The more interesting scenario is the regime that $|I|>n-r$. In this case, if (7) is tight, then one can achieve a non-trivial improvement on communication overhead compared to the naive decoder $D_{I}^{*}$. The remaining questions are that whether (7) is tight, and how to design secret sharing schemes that achieve this bound. We answer these questions in the following two sections.

\section{Construction from Reed-Solomon Code}

In this section we construct capacity-achieving $(n, k, r, z)_{\mathcal{Q}}$ secret sharing schemes that achieve lower bound (7) on communication overhead for all $I$ such that $|I|=n-r$ or $|I|=n$. In other words, the proposed schemes achieve optimal communication bandwidth both in the case that a minimal set of parties are accessible, and in the case that the maximal set of parties are accessible. We note that Shamir's scheme also achieves optimal communication bandwidth when a minimal set of parties are accessible. However, Shamir's scheme is not optimal if more parties are accessible, because regardless of the number of accessible parties, the secret is decoded by selecting only a minimal set of $n-r$ parties and downloading from them. In contrast, our construction enables a more flexible decoding algorithm that can adapt to the number of accessible parties. Specifically, in the case that less than $n$ parties are accessible, decoding is similar to Shamir's scheme. In the case that a maximal set of $n$ parties are accessible, the decoder performs differently by downloading from all $n$ parties in order to reduce communication. We remark that the latter case, i.e., all $n$ parties are accessible, is particularly important, because by Theorem 1 , it corresponds 
to the best case in terms of communication efficiency. Furthermore, it is arguably the most relevant case for the application of distributed storage of secret data, where the storage nodes are usually highly available. Our construction utilizes Reed-Solomon codes and can be efficiently encoded and decoded. As we are mainly interested in constructing capacityachieving schemes, in the remaining part of the paper (Section 4 and 5) we always fix $k=n-r-z$.

\subsection{Encoding}

Recall that $k=n-r-z$ so that the scheme achieves capacity. Let $q>n(k+r)$ be a prime power, and set $\mathcal{Q}=\mathbb{F}_{q}^{k+r}$. Therefore each share of the secret is a length $k+r$ vector over $\mathbb{F}_{q}$. For $j=1, \ldots, n$, denote the $j$-th share by $c_{j}=\left(c_{1, j}, \ldots, c_{k+r, j}\right)$, where $c_{i, j} \in \mathbb{F}_{q}$. The secret $\boldsymbol{m}$ is $k$ symbols over $\mathcal{Q}$ and therefore can be regarded as a length- $k(k+r)$ vector over $\mathbb{F}_{q}$, denoted by $\left(m_{1}, \ldots, m_{k(k+r)}\right)$. In order to satisfy the information-theoretic security requirement $(2)$, the encoder generates randomness that is independent from the secret. Let $\boldsymbol{k}=\left(k_{1}, \ldots, k_{k z}\right) \in \mathbb{F}_{q}^{k z}$ and $\boldsymbol{k}^{\prime}=\left(k_{1}^{\prime}, \ldots, k_{r z}^{\prime}\right) \in \mathbb{F}_{q}^{r z}$ be independently and uniformly distributed. The encoding scheme is linear over $\mathbb{F}_{q}$, and is described by a $(n k+r z) \times n(k+r)$ encoding matrix $G$ over $\mathbb{F}_{q}$ :

$$
\left(c_{1,1}, \ldots, c_{1, n}, \ldots, c_{k+r, 1}, \ldots, c_{k+r, n}\right)=\left(m_{1}, \ldots, m_{k(k+r)}, k_{1}, \ldots, k_{k z}, k_{1}^{\prime}, \ldots, k_{r z}^{\prime}\right) G .
$$

In the following we discuss the construction of $G$ based on a Vandermonde matrix. We start with some notations. Let $\alpha_{1}, \ldots, \alpha_{n(k+r)}$ be distinct non-zero elements of $\mathbb{F}_{q}$, and let $v_{i j}=\alpha_{j}^{i-1}, i=1, \ldots, n k+r z, j=1, \ldots, n(k+r)$, then $V=\left(v_{i j}\right)$ is a Vandermonde matrix of the same size as $G$. Suppose $\boldsymbol{f}=\left(f_{0}, \ldots, f_{d}\right)$ is an arbitrary vector with entries in $\mathbb{F}_{q}$, we denote by $\boldsymbol{f}[x]$ the polynomial $f_{0}+f_{1} x+\ldots+f_{d} x^{d}$ over $\mathbb{F}_{q}$ with indeterminate $x$. We construct a set of polynomials as follows:

$$
\begin{array}{rlrl}
\boldsymbol{f}_{i}[x] & =x^{i-1} & i & =1, \ldots, k n, \\
\boldsymbol{f}_{k n+i}[x] & =x^{i-1} \prod_{j=1}^{k n}\left(x-\alpha_{j}\right) & i & =1, \ldots, r z .
\end{array}
$$

Let $\boldsymbol{f}_{i}, i=1, \ldots, k n+r z$ be the length- $(k n+r z)$ vectors over $\mathbb{F}_{q}$ corresponding to the polynomials. Stack the $\boldsymbol{f}_{i}$ 's to obtain a sqaure matrix of size $(k n+r z)$ :

$$
T=\left(\begin{array}{c}
\boldsymbol{f}_{1} \\
\vdots \\
\boldsymbol{f}_{k n+r z}
\end{array}\right)
$$

Finally, we complete the construction by setting

$$
G=T V
$$


Example 1. Consider the setting that $n=3, r=1, z=1$ and $k=n-r-z=1$. Let $q=7$ and $\mathcal{Q}=\mathbb{F}_{q}^{2}$. Then $\boldsymbol{m}=\left(m_{1}, m_{2}\right), \boldsymbol{k}=\left(k_{1}\right)$ and $\boldsymbol{k}^{\prime}=\left(k_{1}^{\prime}\right)$. Construct a Vandermonde matrix over $\mathbb{F}_{q}$ as

$$
V=\left(\begin{array}{llllll}
1 & 1 & 1 & 1 & 1 & 1 \\
1 & 2 & 3 & 4 & 5 & 6 \\
1 & 4 & 2 & 2 & 4 & 1 \\
1 & 1 & 6 & 1 & 6 & 6
\end{array}\right)
$$

Construct polynomials $\boldsymbol{f}_{1}[x]=1, \boldsymbol{f}_{2}[x]=x, \boldsymbol{f}_{3}[x]=x^{2}$ and

$$
\boldsymbol{f}_{4}[x]=(x-1)(x-2)(x-3)=1+4 x+x^{2}+x^{3} .
$$

Therefore,

$$
T=\left(\begin{array}{l}
\boldsymbol{f}_{1} \\
\boldsymbol{f}_{2} \\
\boldsymbol{f}_{3} \\
\boldsymbol{f}_{4}
\end{array}\right)=\left(\begin{array}{llll}
1 & 0 & 0 & 0 \\
0 & 1 & 0 & 0 \\
0 & 0 & 1 & 0 \\
1 & 4 & 1 & 1
\end{array}\right),
$$

and the encoding matrix is given by

$$
G=T V=\left(\begin{array}{llllll}
1 & 1 & 1 & 1 & 1 & 1 \\
1 & 2 & 3 & 4 & 5 & 6 \\
1 & 4 & 2 & 2 & 4 & 1 \\
0 & 0 & 0 & 6 & 3 & 4
\end{array}\right)
$$

This is exactly the scheme shown in Figure 2b, regarding $k_{2}$ in the figure as $k_{1}^{\prime}$.

The properties of $G$ are discussed in the following lemma.

Lemma 1. Regard $G$ as a block matrix

$$
G=\left(\begin{array}{ll}
G_{11} & G_{12} \\
G_{21} & G_{22}
\end{array}\right)
$$

where $G_{11}$ has size $k n \times k n, G_{12}$ has size $k n \times r n, G_{21}$ has size $r z \times k n$, and $G_{22}$ has size $r z \times r n$. Then,

(i) Any $(n-r)(k+r)$ columns of $G$ are linearly independent.

(ii) $G_{11}$ is a Vandermonde matrix.

(iii) $G_{21}=0$.

(iv) Any rz columns of $G_{22}$ are linearly independent. 
Proof. By construction, the polynomials $\boldsymbol{f}_{i}[x], i=1, \ldots, k n+r z$ have distinct degrees and therefore are linearly independent. Therefore the rows of $T$ are linearly independent and so $T$ is full rank. This implies that the row space of $G$ is the same as the row space of $V$. The row space of $V$ is a linear $(n k+n r, n k+r z)$ MDS $\operatorname{code}^{1}$ because that $V$ is a Vandermonde matrix. Note that $n k+r z=(n-r)(k+r)$, and so the row space of $G$ is a linear $(n k+n r,(n-r)(k+r))$ MDS code. This proves (i).

To prove (ii), note that by (12), the first $k n$ rows of $G$ are exactly the first $k n$ rows of $V$. Therefore $G_{11}$ is a Vandermonde matrix.

To prove (iii), note that by construction the $(i, j)$-th entry of $G_{21}$ equals $\boldsymbol{f}_{k n+i}\left[\alpha_{j}\right]$. By (13), $\alpha_{j}$ is a root of $\boldsymbol{f}_{k n+i}[x]$, for $i=1, \ldots, r z, j=1, \ldots, k n$. Hence $G_{21}=0$.

Finally we prove (iv). By construction the $(i, j)$-th entry of $G_{22}$ equals

$$
\boldsymbol{f}_{k n+i}\left[\alpha_{k n+j}\right]=\alpha_{k n+j}^{i-1} \prod_{l=1}^{k n}\left(\alpha_{k n+j}-\alpha_{l}\right)=\alpha_{k n+j}^{i-1} \boldsymbol{f}^{*}\left[\alpha_{k n+j}\right],
$$

where $\boldsymbol{f}^{*}[x]=\prod_{l=1}^{k n}\left(x-\alpha_{l}\right)$. Since $\alpha_{1}, \ldots, \alpha_{(k+r) n}$ are distinct elements, it follows that $\boldsymbol{f}^{*}\left[\alpha_{k n+j}\right] \neq 0$, for $j=1, \ldots, r n$. Let $1 \leq j_{1}<j_{2}<\ldots<j_{r z} \leq r n$ and consider the submatrix formed by the $j_{1}$-th, $\ldots, j_{r z}$-th columns of $G_{22}$. By (15), the $l$-th column of the submatrix are formed by consecutive powers of $\alpha_{k n+j_{l}}$, scaled by $\boldsymbol{f}^{*}\left[\alpha_{k n+j_{l}}\right]$. Therefore the determinant of the submatrix is $\prod_{l=1}^{r z} \boldsymbol{f}^{*}\left[\alpha_{k n+j_{l}}\right] \prod_{1 \leq u<v \leq r z}\left(\alpha_{k n+j_{v}}-\alpha_{k n+j_{u}}\right) \neq 0$. This shows that any $r z$ columns of $G_{22}$ are linearly independent.

\subsection{Decoding}

We describe the decoding procedure for two cases: 1) $|I|=n$, i.e., all parties are accessible, and 2) $n-r \leq|I|<n$, i.e., some but no more than $r$ parties are inaccessible.

First consider the case that $|I|=n$, i.e., $I=[n]$. In order to decode, for this case it suffices to read and communicate the first $k$ symbols over $\mathbb{F}_{q}$ from each share. Formally, let the ranges of the preprocessing functions be $\mathcal{S}_{i}=\mathbb{F}_{q}^{k}$ and let the preprocessing functions be $E_{i}\left(c_{i}\right)=\left(c_{1, i}, \ldots, c_{k, i}\right)$. Therefore $\boldsymbol{e}_{[n]}=\left(c_{1,1}, \ldots, c_{1, n}, \ldots, c_{k, 1}, \ldots, c_{k, n}\right)$. By Lemma 1.(ii), $G_{11}$ is invertible. Denote the inverse of $G_{11}$ by $G_{11}^{-1}$, then the secret can be recovered by

$$
D_{[n]}\left(\boldsymbol{e}_{[n]}\right)=\boldsymbol{e}_{[n]} G_{11}^{-1} \stackrel{(e)}{=}\left(m_{1}, \ldots, m_{k(k+r)}, k_{1}, \ldots, k_{k z}\right),
$$

where (e) follows from (11) and Lemma 1.(iii). The decoding process involves communicating $k n$ symbols from $\mathbb{F}_{q}$. Recall that all log functions in the paper are base $Q$. The communication overhead is

$$
\mathrm{CO}([n])=n \cdot \log \left(q^{k}\right)-k=\frac{n k}{k+r}-k=\frac{k z}{n-z},
$$

\footnotetext{
${ }^{1}$ In fact this is the Reed-Solomon code.
} 
which achieves the lower bound (7) and therefore is optimal.

Next consider the case that $n-r \leq|I|<n$. In this case we resort to a naive decoding scheme as follows. Select an arbitrary subset $I^{\prime}$ of $I$ of size $n-r$. For $i \in I^{\prime}$, let $\mathcal{S}_{i}=\mathcal{Q}$ and let $E_{i}$ be the identity function. For $i \notin I^{\prime}$, let $\mathcal{S}_{i}$ be the empty set, i.e., these parties do not participate in decoding. Hence, $\boldsymbol{e}_{I^{\prime}}$ is a length- $(n-r)(k+r)$ vector over $\mathbb{F}_{q}$. By Lemma 1.(i), it follows that the corresponding $(n-r)(k+r)$ columns in $G$ are linearly independent and therefore the submatrix formed by these columns is invertible. The secret $\boldsymbol{m}$ can then be recovered by multiplying $\boldsymbol{e}_{I^{\prime}}$ with the inverse. An alternative way to decode the secret is to notice that $G$ is an encoding matrix of a $(n k+n r, n k+r z)$ Reed-Solomon code over $\mathbb{F}_{q}$. Therefore one may employ the standard decoder of Reed-Solomon code to correct any $r(k+r)$ erasures of symbols over $\mathbb{F}_{q}$. Note that for all parties that do not participate in decoding, i.e., for all $i \notin I^{\prime}$, if we regard their shares as being erased, then there are exactly $r(k+r)$ erasures of symbols over $\mathbb{F}_{q}$, and therefore can be corrected. Either way, the decoding process involves communicating $n k+r z$ symbols of $\mathbb{F}_{q}$. The communication overhead during decoding is

$$
\mathrm{CO}(I)=\frac{n k+r z}{k+r}-k=z,
$$

which achieves the lower bound (7) if and only if $|I|=n-r$.

\subsection{Analysis}

Theorem 2. The encoding scheme constructed in Section 4.1 is a capacity-achieving $(n, k, r, z)_{\mathcal{Q}}$ secret sharing scheme. The communication overhead of the corresponding decoding scheme described in Section 4.2 is

$$
\mathrm{CO}(I)=\left\{\begin{array}{cc}
z & |I|<n \\
\frac{k}{n-z} z & |I|=n
\end{array}\right.
$$

which is optimal for $|I|=n$ and $|I|=n-r$.

Proof. We need to verify that the encoding scheme meets the reliability requirement and the security requirement of a secret sharing scheme, formally defined in Definition 1. Explicit decoding schemes are discussed in Section 4.2 and therefore the reliability requirement is met. We only need to show that the encoding scheme is also secure. To this end, we first show that $H\left(\boldsymbol{k}, \boldsymbol{k}^{\prime} \mid \boldsymbol{c}_{I}, \boldsymbol{m}\right)=0$, for all $I$ such that $|I|=z$. In other words, the random symbols generated by the encoder are completely determined by $\boldsymbol{c}_{I}$ and the secret. Denote the submatrix formed by the first $k(k+r)$ rows of $G$ by $G_{\text {top }}$ and the submatrix formed by the remaining $(k+r) z$ rows of $G$ by $G_{\text {low }}$. Consider any $I=\left\{i_{1}, \ldots, i_{z}\right\}$, and let $\boldsymbol{c}_{I}=\left(c_{1, i_{1}}, \ldots, c_{1, i_{z}}, \ldots, c_{k+r, i_{1}}, \ldots, c_{k+r, i_{z}}\right)$. It then follows from (11) that

$$
\boldsymbol{c}_{I}=\left(m_{1}, \ldots, m_{k(r+k)}\right) G_{\mathrm{top}, I}+\left(k_{1}, \ldots, k_{k z}, k_{1}^{\prime}, \ldots, k_{r z}^{\prime}\right) G_{\mathrm{low}, I},
$$


where $G_{\mathrm{top}, I}$ is the submatrix formed by the subset of columns in $\{i+j \mid i \in I, j=$ $0, n, \ldots,(k+r-1) n\}$ of $G_{\mathrm{top}}$, and $G_{\mathrm{low}, I}$ is the submatrix formed by the same subset of columns of $G_{\text {low }}$. Therefore, written concisely,

$$
\left(\boldsymbol{k} \boldsymbol{k}^{\prime}\right) G_{\mathrm{low}, I}=\boldsymbol{c}_{I}-\boldsymbol{m} G_{\mathrm{top}, I} .
$$

To study the rank of $G_{\text {low }, I}$, note that it is a square matrix of size $(k+r) z$, and we regard it as a block matrix

$$
G_{\mathrm{low}, I}=\left(\begin{array}{ll}
G_{11}^{\prime} & G_{12}^{\prime} \\
G_{21}^{\prime} & G_{22}^{\prime}
\end{array}\right)
$$

where $G_{11}^{\prime}$ has size $k z \times k z, G_{12}^{\prime}$ has size $k z \times r z, G_{21}^{\prime}$ has size $r z \times k z$ and $G_{22}^{\prime}$ has size $r z \times r z$. By Lemma 1.(ii), $G_{11}^{\prime}$ is a block of a Vandermonde matrix and therefore is invertible. By Lemma 1.(iii), $G_{21}^{\prime}=0$. Denote $\boldsymbol{c}_{I}-\boldsymbol{m} G_{\mathrm{top}, I}$ by $\left(u_{1}, \ldots, u_{(k+r) z}\right)$, then the above two facts together with (16) imply that

$$
\boldsymbol{k}=\left(u_{1}, \ldots, u_{k z}\right) G_{11}^{\prime-1}
$$

Therefore $\boldsymbol{k}$ is a deterministic function of $\boldsymbol{m}$ and $\boldsymbol{c}_{I}$. It follows from (16) that

$$
\boldsymbol{k}^{\prime} G_{22}^{\prime}=\left(u_{k z+1}, \ldots, u_{(k+r) z}\right)-\boldsymbol{k} G_{12}^{\prime} .
$$

By Lemma 1.(iv), $G_{22}^{\prime}$ is invertible and therefore

$$
\boldsymbol{k}^{\prime}=\left(\left(u_{k z+1}, \ldots, u_{(k+r) z}\right)-\boldsymbol{k} G_{12}^{\prime}\right) G_{22}^{\prime-1} .
$$

This shows that $\boldsymbol{k}^{\prime}$ is a deterministic function of $\boldsymbol{k}, \boldsymbol{c}_{I}$ and $\boldsymbol{m}$, and so

$$
H\left(\boldsymbol{k}, \boldsymbol{k}^{\prime} \mid \boldsymbol{c}_{I}, \boldsymbol{m}\right)=0
$$

It then follows that,

$$
\begin{aligned}
H(\boldsymbol{m})-H\left(\boldsymbol{m} \mid \boldsymbol{c}_{I}\right) & =I\left(\boldsymbol{m} ; \boldsymbol{c}_{I}\right) \\
& =H\left(\boldsymbol{c}_{I}\right)-H\left(\boldsymbol{c}_{I} \mid \boldsymbol{m}\right) \\
& \stackrel{(f)}{\leq} z-H\left(\boldsymbol{c}_{I} \mid \boldsymbol{m}\right) \\
& \stackrel{(g)}{=} z-H\left(\boldsymbol{c}_{I} \mid \boldsymbol{m}\right)+H\left(\boldsymbol{c}_{I} \mid \boldsymbol{m}, \boldsymbol{k}, \boldsymbol{k}^{\prime}\right) \\
& =z-I\left(\boldsymbol{c}_{I} ; \boldsymbol{k}, \boldsymbol{k}^{\prime} \mid \boldsymbol{m}\right) \\
& =z-H\left(\boldsymbol{k}, \boldsymbol{k}^{\prime} \mid \boldsymbol{m}\right)+H\left(\boldsymbol{k}, \boldsymbol{k}^{\prime} \mid \boldsymbol{c}_{I}, \boldsymbol{m}\right) \\
& \stackrel{(h)}{=} z-H\left(\boldsymbol{k}, \boldsymbol{k}^{\prime} \mid \boldsymbol{m}\right) \\
& \stackrel{(i)}{=} z-H\left(\boldsymbol{k}, \boldsymbol{k}^{\prime}\right) \\
& \stackrel{(j)}{=} z-z=0,
\end{aligned}
$$


where (f) is due to $|I|=z$; (g) is due to the fact that $\boldsymbol{c}_{I}$ is a function of $\boldsymbol{m}, \boldsymbol{k}$ and $\boldsymbol{k}^{\prime}$; (h) is due to (20); (i) is due to the fact that $\boldsymbol{k}, \boldsymbol{k}^{\prime}$ are independent of $\boldsymbol{m}$; and (j) follows from the fact that $\boldsymbol{k}, \boldsymbol{k}^{\prime}$ are uniformly distributed. Therefore $H(\boldsymbol{m})=H\left(\boldsymbol{m} \mid \boldsymbol{c}_{I}\right)$ and the security requirement is met. This completes the proof that the encoding scheme is a valid secret sharing scheme. The scheme is capacity-achieving because $k=n-r-z$.

The statement on the communication overhead of the decoder is evident from the discussion in Section 4.2.

Theorem 2 shows that the proposed secret sharing scheme is optimal in terms of storage usage and is optimal in terms of best-case (i.e., $|I|=n$ ) communication overhead. Note that the optimal communication overhead when $|I|=n$ is only a fraction $\frac{k}{n-z}=\frac{n-r-z}{n-z}$ of the optimal communication overhead when $|I|=n-r$.

We conclude this section by remarking on some other important advantages and features of the construction. Firstly, the scheme also achieves the optimal number of symbol-reads from disks in decoding if $|I|=n$ or $|I|=n-r$. To see this, notice that the lower bound (7) on communication overhead is also a lower bound on the number of $Q$-ary symbols that need to be read from disks during decoding. The number of symbol-reads in the proposed scheme equals to the amount of communication. Therefore it achieves the lower bound and hence is optimal. Secondly, by involving more than the minimum number of disks for decoding, the scheme balances the load at the disks and achieves a higher degree of parallelization. Thirdly, the encoding and decoding schemes may be implemented as the encoding and decoding schemes for Reed-Solomon codes, and therefore are efficient and practical. Fourthly, the preprocessing functions only rely on $|I|$ instead of $I$, further simplifying implementation. Fiffthly, our construction is flexible in the parameters, i.e., it works for arbitrary values of $n, r$ and $z$. Finally, in the scheme all operations are performed over the field $\mathbb{F}_{q}$, where $q>n(k+r)$. This requirement on the field size can be relaxed in the following simple way. Let $\beta$ be the greatest common divisor of $k$ and $r$, then instead of choosing $\mathcal{Q}$ to be $\mathbb{F}_{q}^{k+r}$, we can let $\mathcal{Q}=\mathbb{F}_{q}^{\frac{k}{\beta}+\frac{r}{\beta}}, \boldsymbol{m}=\left(m_{1}, \ldots, m_{\frac{k(k+r)}{\beta}}\right), \boldsymbol{k}=\left(k_{1}, \ldots, k_{\frac{k z}{\beta}}\right)$ and $\boldsymbol{k}^{\prime}=\left(k_{1}^{\prime}, \ldots, k_{\frac{r z}{\beta}}^{\prime}\right)$. The resulting scheme is also a capacity-achieving $(n, k, r, z)_{\mathcal{Q}}$ secret sharing scheme with the same communication overhead function as the original scheme. For this modified construction, it is sufficient to choose any field size $q>n \frac{k+r}{\beta}$.

\section{Secret Sharing Schemes from Random Codes}

In the previous section we constructed practical secret sharing schemes that achieve the lower bound (7) on communication overhead for all $I$ such that $|I|=n$ and $|I|=n-r$. The remaining question is whether the lower bound is tight for all $I$. In this section we answer this question affirmatively by designing a secret sharing scheme that achieves the bound for all $I$. The scheme utilizes random linear codes. The scheme meets the security requirement 
(2) deterministically, and meets the reliability requirement (1) with high probability as the field size grows to infinity.

\subsection{Encoding}

Again we fix $k=n-r-z$ so that the scheme achieves capacity. Let $q$ be a prime power, and let $N$ be the least common multiple of $\{n-z-r, n-z-r+1, \ldots, n-z\}$, i.e., $N$ is the smallest positive integer that is divisible by all elements of the set. Set $\mathcal{Q}=\mathbb{F}_{q}^{N(k+r)}$. Therefore each share of the secret is a length $N(k+r)$ vector over $\mathbb{F}_{q}$. For $j=1, \ldots, n$, denote the $j$-th share by $c_{j}=\left(c_{1, j}, \ldots, c_{N(k+r), j}\right)$, where $c_{i, j} \in \mathbb{F}_{q}$. The secret $\boldsymbol{m}$ consists of $k$ symbols over $\mathcal{Q}$ and is regarded as a length- $N k(k+r)$ vector over $\mathbb{F}_{q}$, denoted by $\left(m_{1}, \ldots, m_{N k(k+r)}\right)$. The encoder generates uniformly distributed random vectors $\boldsymbol{k}=\left(k_{1}, \ldots, k_{N k z}\right) \in \mathbb{F}_{q}^{N k z}$ and $\boldsymbol{k}^{\prime}=\left(k_{1}^{\prime}, \ldots, k_{N r z}^{\prime}\right) \in \mathbb{F}_{q}^{N r z}$, independently from $\boldsymbol{m}$. The encoding scheme is described by a set of $N(k n+r z) \times n$ encoding matrices $G_{i}, i=1, \ldots, N(k+r)$ over $\mathbb{F}_{q}$, such that

$$
\left(c_{i, 1}, \ldots, c_{i, n}\right)=\left(\boldsymbol{m} \boldsymbol{k} \boldsymbol{k}^{\prime}\right) G_{i}, \quad i=1, \ldots, N(k+r) .
$$

Intuitively, if the $c_{u, v}$ 's are arranged into a matrix (refer to Figure $2 \mathrm{~b}$ for an example) then $G_{i}$ is the encoding matrix for the $i$-th row. We next describe the construction of the $G_{i}$ matrices. For $i=1, \ldots, N k$, let the first $N k(k+r)$ rows of $G_{i}$ be a random matrix, let the next $N k z$ rows of $G_{i}$ be a Vandermonde matrix, and let the remaining $N r z$ rows of $G_{i}$ be zero. Formally, for $i=1, \ldots, N k$,

$$
G_{i}=\left(\begin{array}{c}
R_{i} \\
V_{i} \\
\mathbf{0}
\end{array}\right),
$$

where $R_{i} \in \mathbb{F}_{q}^{N k(k+r) \times n}$ is a random matrix with entries i.i.d. uniformly distributed over $\mathbb{F}_{q}$, and $V_{i} \in \mathbb{F}_{q}^{N k z \times n}$ is a Vandermonde matrix, i.e., the $(u, v)$-th entry of $V_{i}$ equals $\alpha_{v, i}^{u-1}$. Here $\alpha_{v, i}$ are distinct non-zero elements of $\mathbb{F}_{q}$, for $i=1, \ldots, N(k+r)$, and $v=1, \ldots, n$.

For $i=1, \ldots, N r$, let the first $N k n+(i-1) z$ rows of $G_{N k+i}$ be a random matrix, let the next $z$ rows of $G_{N k+i}$ be a Vandermonde matrix, and let the remaining $(N r-i) z$ rows of $G_{N k+i}$ be zero. Formally, for $i=1, \ldots, N r$,

$$
G_{N k+i}=\left(\begin{array}{c}
R_{N k+i} \\
V_{N k+i} \\
\mathbf{0}
\end{array}\right),
$$

where $R_{N k+i} \in \mathbb{F}_{q}^{(N k n+(i-1) z) \times n}$ is a random matrix with entries i.i.d. uniformly distributed over $\mathbb{F}_{q}$, and $V_{N k+i} \in \mathbb{F}_{q}^{z \times n}$ is a Vandermonde matrix, i.e., the $(u, v)$-th entry of $V_{N k+i}$ equals $\alpha_{v, N k+i}^{u-1}$. This completes the encoding scheme. The structure of the whole encoding matrix $\left(G_{1}, \ldots, G_{N(k+r)}\right)$ is illustrated in Figure 3. 


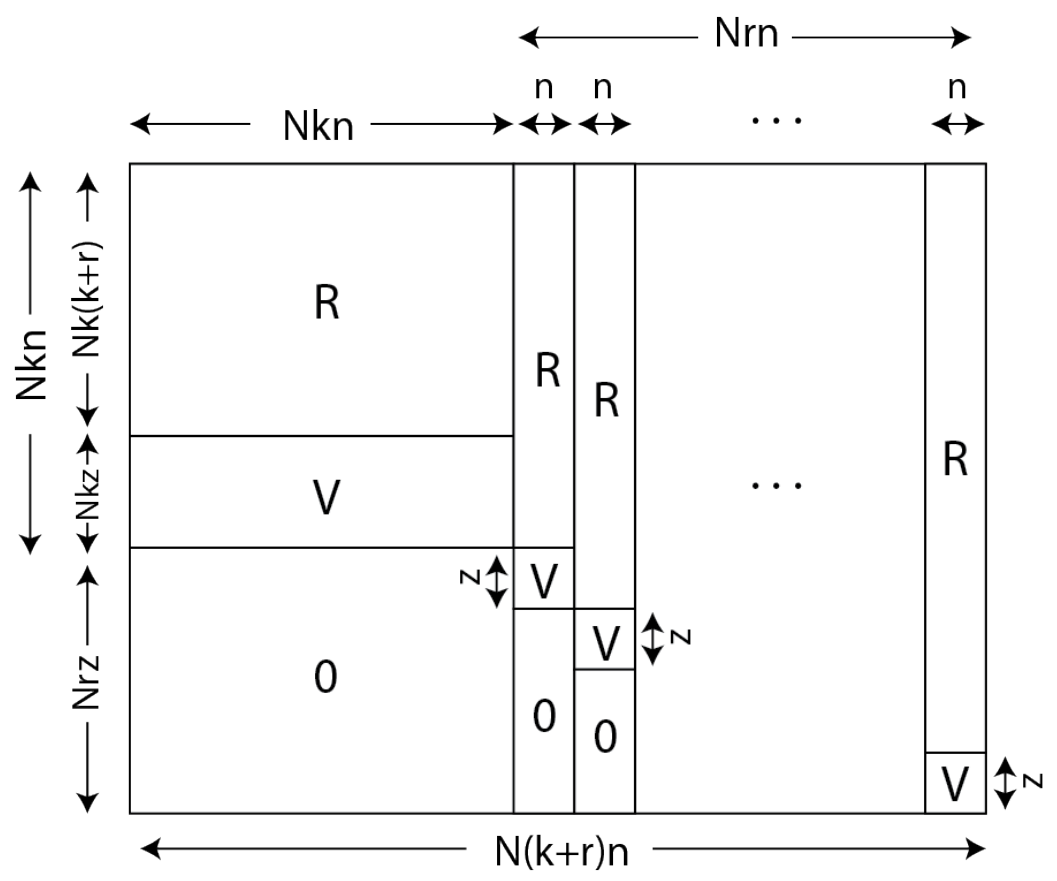

Figure 3: Blockwise structure of the matrix $\left(G_{1}, \ldots, G_{N(k+r)}\right)$. Blocks of random matrices are labelled by $\mathrm{R}$, blocks of Vandermonde matrices are labelled by $\mathrm{V}$, and blocks of zero matrices are labelled by 0 .

The following result shows that the scheme meets the security requirement deterministically, due to the Vandermonde matrices embedded in the $G_{i}$ 's.

Theorem 3. The encoding scheme constructed in this section is secure, i.e., $H\left(\boldsymbol{m} \mid \boldsymbol{c}_{I}\right)=$ $H(\boldsymbol{m})$, for all $I$ such that $|I|=z$.

Proof. Consider any $I$ such that $|I|=z$. As in Theorem 2, we first show that $H\left(\boldsymbol{k}, \boldsymbol{k}^{\prime} \mid \boldsymbol{c}_{I}, \boldsymbol{m}\right)=$ 0 . Denote by $G_{i, I}, R_{i, I}$ and $V_{i, I}$ the submatrix formed by the set of columns in $I$ of $G_{i}, R_{i}$ and $V_{i}$, respectively. Let

$$
\begin{aligned}
G_{1 \rightarrow N k, I} & =\left(\begin{array}{llll}
G_{1, I} & \ldots & G_{N k, I}
\end{array}\right) \\
R_{1 \rightarrow N k, I} & =\left(\begin{array}{lll}
R_{1, I} & \ldots & R_{N k, I}
\end{array}\right) \\
V_{1 \rightarrow N k, I} & =\left(\begin{array}{llll}
V_{1, I} & \ldots & V_{N k, I}
\end{array}\right) .
\end{aligned}
$$

Denote for short $\mathfrak{c}_{i}=\left(c_{i, j}\right)_{j \in I}$, then by (22), it follows that

$$
\left(\boldsymbol{m} \boldsymbol{k} \boldsymbol{k}^{\prime}\right) G_{1 \rightarrow N k, I}=\left(\begin{array}{l}
\boldsymbol{k} \boldsymbol{k} \boldsymbol{k}^{\prime}
\end{array}\right)\left(\begin{array}{c}
R_{1 \rightarrow N k, I} \\
V_{1 \rightarrow N k, I} \\
\mathbf{0}
\end{array}\right)
$$




$$
=\left(\mathfrak{c}_{1}, \ldots, \mathfrak{c}_{N k}\right) .
$$

Notice that $V_{1 \rightarrow N k, I}$ is a $N k z \times N k z$ square Vandermonde matrix. Therefore it is invertible and

$$
\boldsymbol{k}=\left(\left(\mathfrak{c}_{1}, \ldots, \mathfrak{c}_{N k}\right)-\boldsymbol{m} R_{1 \rightarrow N k, I}\right) V_{1 \rightarrow N k, I}^{-1}
$$

Hence $H\left(\boldsymbol{k} \mid \boldsymbol{c}_{I}, \boldsymbol{m}\right)=0$. Then by $(22)$, it follows that

$$
\left(\boldsymbol{m} \boldsymbol{k}\left|k_{1}^{\prime}, \ldots, k_{z}^{\prime}\right| k_{z+1}^{\prime}, \ldots, k_{N r z}^{\prime}\right)\left(\frac{R_{N k+1, I}}{\frac{V_{N k+1, I}}{\mathbf{0}}}\right)=\mathfrak{c}_{N k+1} .
$$

Notice that $V_{N k+1, I}$ is a $z \times z$ square Vandermonde matrix. Therefore it is invertible and

$$
\left(k_{1}^{\prime}, \ldots, k_{z}^{\prime}\right)=\left(\mathfrak{c}_{N k+1}-(\boldsymbol{m} \boldsymbol{k}) R_{N k+1, I}\right) V_{N k+1, I}^{-1} .
$$

Hence $H\left(k_{1}^{\prime}, \ldots, k_{z}^{\prime} \mid \boldsymbol{k}, \boldsymbol{c}_{I}, \boldsymbol{m}\right)=0$. Similarly, we can show that for $i=1, \ldots, N r$

$$
H\left(k_{(i-1) z+1}^{\prime}, \ldots, k_{i z}^{\prime} \mid k_{1}^{\prime}, \ldots, k_{(i-1) z}^{\prime}, \boldsymbol{k}, \boldsymbol{c}_{I}, \boldsymbol{m}\right)=0 .
$$

Therefore by the chain rule,

$$
\begin{aligned}
H\left(\boldsymbol{k}, \boldsymbol{k}^{\prime} \mid \boldsymbol{c}_{I}, \boldsymbol{m}\right) & =H\left(\boldsymbol{k} \mid \boldsymbol{c}_{I}, \boldsymbol{m}\right)+\sum_{i=1}^{N r} H\left(k_{(i-1) z+1}^{\prime}, \ldots, k_{i z}^{\prime} \mid k_{1}^{\prime}, \ldots, k_{(i-1) z}^{\prime}, \boldsymbol{k}, \boldsymbol{c}_{I}, \boldsymbol{m}\right) \\
& =0 .
\end{aligned}
$$

Provided that (25) is true, we can then follow exactly the same argument as (21) in the proof of Theorem 2, to show that $H\left(\boldsymbol{m} \mid \boldsymbol{c}_{I}\right)=H(\boldsymbol{m})$. This completes the proof.

\subsection{Decoding}

We describe the decoding scheme for any $I$ such that $|I| \geq n-r$. Let

$$
d \triangleq \frac{N k(n-|I|)}{|I|-z}
$$

then note that $d$ is an integer because $|I|-z$ divides $N$ and that $d$ is the solution to the equation $(N k+d)|I|=N k n+d z$. In order to decode, it suffices to read and communicate the first $N k+d$ symbols over $\mathbb{F}_{q}$ from each available share. Intuitively, by reading the first $N k+d$ symbols from each available share, we have a system of $(N k+d)|I|$ equations. On the other hand, the variables involved in these equations are $m_{1}, \ldots, m_{N k(k+r)}, k_{1}, \ldots, k_{N k z}$ and $k_{1}^{\prime}, \ldots, k_{d z}^{\prime}$, i.e., the total number of variables is $N k n+d z$. Because $d$ is the solution 
to $(N k+d)|I|=N k n+d z$, the number of equations in the system equals the number of variables, and is uniquely solvable if the equations are linearly independent.

Formally, let $\mathcal{S}_{i}=\mathbb{F}_{q}^{N k+d}$, and let $E_{i}\left(c_{i}\right)=\left(c_{1, i}, \ldots, c_{N k+d, i}\right)$. Denote for short that $\mathfrak{c}_{i}=\left(c_{i, j}\right)_{j \in I}$, and denote the submatrix formed by the set of columns in $I$ of $G_{i}$ by $G_{i, I}$. Then it follows from (22) that,

$$
\boldsymbol{e}_{I}=\left(\mathfrak{c}_{1}, \ldots, \mathfrak{c}_{N k+d}\right)=\left(\boldsymbol{m} \boldsymbol{k} \boldsymbol{k}^{\prime}\right)\left(G_{1, I}, \ldots, G_{N k+d, I}\right) .
$$

By construction $(23)$ and $(24)$, the last $(N r-d) z$ rows of the matrices $G_{1, I}, \ldots, G_{N k+d, I}$ are all zeros. Therefore we may delete the last $(N r-d) z$ rows from $G_{1, I}, \ldots, G_{N k+d, I}$ and denote by $\left(G_{1, I}^{*}, \ldots, G_{N k+d, I}^{*}\right)$ the corresponding trimmed matrix. It then follows that,

$$
\boldsymbol{e}_{I}=\left(\mathfrak{c}_{1}, \ldots, \mathfrak{c}_{N k+d}\right)=\left(\boldsymbol{m} \boldsymbol{k} k_{1}^{\prime}, \ldots, k_{d z}^{\prime}\right)\left(G_{1, I}^{*}, \ldots, G_{N k+d, I}^{*}\right) .
$$

It is now evident that if the matrix $\left(G_{1, I}^{*}, \ldots, G_{N k+d, I}^{*}\right)$ has full row rank, then it is right invertible and the secret can be recovered. The following result shows that the matrix indeed has full row rank with high probability.

Theorem 4. For any $I$ such that $|I| \geq n-r,\left(G_{1, I}^{*}, \ldots, G_{N k+d, I}^{*}\right)$ has full row rank with probability at least $1-\frac{1}{q-1}$, over the distribution of the random matrices $R_{1}, \ldots, R_{N k+d}$.

Proof. Note that $\left(G_{1, I}^{*}, \ldots, G_{N k+d, I}^{*}\right)$ has size $(N k n+d z) \times(N k+d)|I|$. By the definition of $d$, it follows that $N k n+d z=(N k+d)|I|$ and therefore the matrix is square. Hence it suffices to show the matrix has full column rank and in the following we show the columns of the matrix are linearly independent with high probability.

The first $N k z$ columns of $\left(G_{1, I}^{*}, \ldots, G_{N k, I}^{*}\right)$ are linearly independent because by (23), the $(N k n-N k z+1)$-th row to the $N k n$-th row form a Vandermonde matrix. Denote the $i$-th column of $\left(G_{1, I}^{*}, \ldots, G_{N k+d, I}^{*}\right)$ by $\boldsymbol{g}_{i}$. We first study the probability that the $\boldsymbol{g}_{N k z+i}$ is in the linear span of all the previous columns, i.e., $\operatorname{span}\left[\boldsymbol{g}_{1}, \ldots, \boldsymbol{g}_{N k z+i-1}\right]$, for $i=1, \ldots, N k(|I|-z)$. Consider the sum of vectors $\boldsymbol{g}^{*}=\sum_{l=1}^{N K z+i-1} \gamma_{l} \boldsymbol{g}_{l}$. Fixing $\gamma_{1}, \ldots, \gamma_{i-1}$ to be arbitrary values in $\mathbb{F}_{q}$, then there is a unique tuple $\left(\gamma_{l}\right)_{l=i}^{N K z+i-1}$ such that $\boldsymbol{g}^{*}$ agrees with $\boldsymbol{g}_{N K z+i}$ in the $(N k n-N k z+1)$-th to $N k n$-th entries. Therefore there are $q^{i-1}$ different ways to linearly combine $\boldsymbol{g}_{1}, \ldots, \boldsymbol{g}_{N k z+i-1}$, such that in the resulting sum vector, the $(N k n-N k z+1)$ th to $N k n$-th entries are equal to the corresponding entries of $\boldsymbol{g}_{N k z+i}$. Because the first $N k(n-z)$ entries of $\boldsymbol{g}_{N k z+i}$ are i.i.d. uniformly distributed, it follows that

$$
\operatorname{Pr}\left\{\boldsymbol{g}_{N k z+i} \in \operatorname{span}\left[\boldsymbol{g}_{1}, \ldots, \boldsymbol{g}_{N k z+i-1}\right]\right\} \leq \frac{q^{i-1}}{q^{N k(n-z)}}, \quad i=1, \ldots, N k(|I|-z)
$$

We next study the probability that $\boldsymbol{g}_{N k|I|+i}$ is in $\operatorname{span}\left[\boldsymbol{g}_{1}, \ldots, \boldsymbol{g}_{N k|I|+i-1}\right]$. Consider arbitrary $N k \leq j \leq N k+d-1$. By construction (24), $\boldsymbol{g}_{j|I|+i} \notin \operatorname{span}\left[\boldsymbol{g}_{1}, \ldots, \boldsymbol{g}_{j|I|+i-1}\right]$, for $1 \leq i \leq z$, due to the Vandermonde matrix $V_{N k+j}$. Now consider $\boldsymbol{g}_{j|I|+i}$ with $z+1 \leq i \leq|I|$. There are $q^{j|I|+i-z-1}$ different ways to linearly combine $\boldsymbol{g}_{1}, \ldots, \boldsymbol{g}_{j|I|+i-1}$, such that in the 
resulting sum vector, the $(N k n+(j-N k) z+1)$-th to $(N k n+(j-N k) z+z)$-th entries are equal to the corresponding entries in $\boldsymbol{g}_{j|I|+i}$. Note that the first $N k n+(j-N k) z$ entries of $\boldsymbol{g}_{j|I|+i}$ are i.i.d. uniformly distributed. Therefore, for $N k \leq j \leq N k+d-1$ and $z+1 \leq i \leq|I|$, it follows that

$$
\operatorname{Pr}\left\{\boldsymbol{g}_{j|I|+i} \in \operatorname{span}\left[\boldsymbol{g}_{1}, \ldots, \boldsymbol{g}_{j|I|+i-1}\right]\right\} \leq \frac{q^{j|I|+i-z-1}}{q^{N k n+(j-N k) z}} .
$$

Hence, by the union bound

$$
\begin{aligned}
& \operatorname{Pr}\left\{\left(G_{1, I}^{*}, \ldots, G_{N k+d, I}^{*}\right) \text { singular }\right\} \\
& \leq \sum_{i=1}^{N k(|I|-z)} \operatorname{Pr}\left\{\boldsymbol{g}_{N k z+i} \text { l.d. }^{2}\right\}+\sum_{j=N k}^{N k+d-1} \sum_{i=z+1}^{|I|} \operatorname{Pr}\left\{\boldsymbol{g}_{j|I|+i} \text { l.d. }\right\} \\
& \stackrel{(k)}{\leq} \sum_{i=1}^{N k(|I|-z)} \frac{q^{i-1}}{q^{N k(n-z)}}+\sum_{j=N k}^{N k+d-1} \sum_{i=z+1}^{|I|} \frac{q^{j|I|+i-z-1}}{q^{N k n+(j-N k) z}} \\
& =\sum_{i=N k(z-n)}^{N k(|I|-n)-1} q^{i}+\sum_{j=N k}^{N k+d-1} \sum_{i=z+1}^{|I|} \frac{q^{j|I|+i-z-1}}{q^{N k n+(j-N k) z}} \\
& =\sum_{i=N k(z-n)}^{N k(|I|-n)-1} q^{i}+\sum_{j=N k}^{N k+d-1} \sum_{i=0}^{|I|-z-1} q^{j(|I|-z)+i+N k(z-n)} \\
& =\sum_{i=N k(z-n)}^{N k(|I|-n)-1} q^{i}+q^{N k(z-n)} \sum_{j=N k}^{N k+d-1|I|-z-1} \sum_{i=0}^{j(|I|-z)+i} \\
& =\sum_{i=N k(z-n)}^{N k(|I|-n)-1} q^{i}+q^{N k(z-n)} \sum_{i=N k(|I|-z)}^{(N k+d)(|I|-z)-1} q^{i} \\
& N k(|I|-n)-1 \quad(N k+d)|I|-N k n-d z-1 \\
& =\sum_{i=N k(z-n)} q^{i}+\sum_{i=N k(|I|-n)} q^{i} \\
& \stackrel{(l)}{=} \sum_{i=N k(z-n)}^{N k(|I|-n)-1} q^{i}+\sum_{i=N k(|I|-n)}^{-1} q^{i} \\
& <\sum_{i=-\infty}^{-1} q^{i}=\frac{1}{q-1}
\end{aligned}
$$

where $(k)$ is due to $(27)$ and $(28)$, and $(l)$ is due to (26). This completes the proof.

\footnotetext{
${ }^{2}$ Linearly dependent on the set of columns to the left.
} 
The following result summarizes the properties of scheme.

Corollary 1. The encoding scheme constructed in Section 5.1 is a capacity-achieving $(n, k, r, z)_{\mathcal{Q}}$ secret sharing scheme with high probability. Specifically, the scheme meets the security requirement deterministically, and meets the reliability requirement with probability at least $1-\frac{2^{n}}{q-1}$, over the distribution of the random matrices $R_{1}, \ldots, R_{N k+d}$. The communication overhead of the corresponding decoding scheme described in Section 5.2 achieves equality in the lower bound (7), for all I such that $|I| \geq n-r$.

Proof. The scheme achieves capacity because $k=n-r-z$. By Theorem 3, the scheme meets the security requirement. By Theorem 4 and the union bound, the scheme meets the reliability requirement with probability at least $1-\sum_{i=0}^{r}\left(\begin{array}{c}n \\ i\end{array}\right) \frac{1}{q-1} \geq 1-\frac{2^{n}}{q-1}$.

Consider any $I$ such that $|I| \geq n-r$. In order to decode, a number of $(N k+d)|I|$ symbols over $\mathbb{F}_{q}$ are communicated. Therefore the communication overhead is

$$
\begin{aligned}
\mathrm{CO}(I) & =\frac{(N k+d)|I|-N k(k+r)}{N(k+r)} \\
& =\frac{N k n+d z-N k(k+r)}{N(k+r)} \\
& =\frac{N k z+d z}{N(k+r)}=\frac{z\left(N k+\frac{N k(n-|I|)}{|I|-z}\right)}{N(k+r)} \\
& =\frac{N k(n-z) z}{(|I|-z) N(k+r)}=\frac{k z}{|I|-z},
\end{aligned}
$$

which achieves equality in (7).

\section{Conclusions}

In this paper we introduce the communication efficiency of secret sharing schemes. We prove an information-theoretic lower bound on the amount of information to be communicated during decoding, given a set of accessible parties $I \subset\{1, \ldots, n\}$. We prove the tightness of the lower bound by designing secret sharing schemes from random linear codes, such that the schemes achieve the lower bound for all $I$. We also construct computationally efficient secret sharing schemes from Reed-Solomon codes that achieves the bound when $|I|=n$ or $|I|=n-r$. In the application of secure distributed storage, the proposed communication efficient secret sharing schemes also improve disk access efficiency. There are a number of open problems: 1) how to construct computationally efficient secret sharing schemes (with small field size) that achieve the lower bound on communication for all $I$ ? 2) how to generalize the results to other (non-threshold) access structures? and 3) in the application of secure distributed storage, how to construct storage codes that are communication efficient in terms of both decoding and repair? 


\section{References}

[1] A. Beimel, "Secret-sharing schemes: a survey," in Coding and Cryptology, Berlin, Heidelberg, 2011, pp. 11-46.

[2] G. R. Blakley, "Safeguarding cryptographic keys," in AFIPS, 1979, pp. 313-317.

[3] M. Blaum, J. Bruck, and A. Vardy, "MDS array codes with independent parity symbols," IEEE Transactions on Information Theory, vol. 42, no. 2, pp. 529-542, 1996.

[4] M. Blaum, J. Brady, J. Bruck, and J. Menon, "EVENODD: an efficient scheme for tolerating double disk failures in RAID architectures," IEEE Transactions on Computers, vol. 44, pp. 192-202, 1995.

[5] H.-Y. Chien, J.-K. Jan, and Y.-M. Tseng, "A practical (t,n) multi-secret sharing scheme," IEICE Transactions on Fundamentals of Electronics, Communications and Computer Sciences, vol. E83-A, 2000.

[6] A. G. Dimakis, K. Ramchandran, Y. Wu, and C. Suh, "A survey on network codes for distributed storage," Proceedings of the IEEE, vol. 99, no. 3, pp. 476-489, Mar. 2011.

[7] M. Ito, A. Saito, and T. Nishizeki, "Multiple assignment scheme for sharing secret," Journal of Cryptology, vol. 6, no. 1, pp. 15-20, Mar. 1993.

[8] M. Karchmer and A. Wigderson, "On span programs," in Structure in Complexity Theory Conference, 1993., Proceedings of the Eighth Annual, 1993, pp. 102-111.

[9] E. D. Karnin, J. W. Greene, and M. E. Hellman, "On secret sharing systems," IEEE Transactions on Information Theory, vol. 29, no. 1, pp. 35-41, Jan. 1983.

[10] C.-P. Lai and C. Ding, "Several generalizations of Shamir's secret sharing scheme," Int. J. Found. Comput. Sci., 2004.

[11] R. J. McEliece and D. V. Sarwate, "On sharing secrets and Reed-Solomon codes," Communications of the ACM, vol. 24, pp. 1-2, 1981.

[12] K. V. Rashmi, N. B. Shah, and P. V. Kumar, "Optimal exact-regenerating codes for distributed storage at the MSR and MBR points via a product-matrix construction," IEEE Transactions on Information Theory, vol. 57, no. 8, pp. 5227-5239, 2011.

[13] A. Shamir, "How to share a secret," Communications of the ACM, p. 7, 1979.

[14] I. Tamo, Z. Wang, and J. Bruck, "Zigzag codes: MDS array codes with optimal rebuilding," IEEE Transactions on Information Theory, vol. 59, no. 3, pp. 1597-1616, 2013. 
[15] C.-C. Yang, T.-Y. Chang, and M.-S. Hwang, "A (t,n) multi-secret sharing scheme," Applied Mathematics and Computation, vol. 151, no. 2, pp. 483-490, Apr. 2004. 